\title{
Fast Diffusion and Void Formation in a Two-Segment Copper-Tin Lead-Free Nanowire System with One-Dimensional Confinement
}

\author{
Fan Gao', Zhenyu Liu², Guangwen Zhou' ${ }^{3}$, Judith C. Yang ${ }^{2}$, and Zhiyong Gu ${ }^{1, *}$ \\ ${ }^{1}$ Department of Chemical Engineering, University of Massachusetts Lowell, Lowell, MA 01854, USA \\ ${ }^{2}$ Department of Chemical and Petroleum Engineering, University of Pittsburgh, Pittsburgh, PA 15261, USA \\ ${ }^{3}$ Department of Mechanical Engineering, Binghamton University, State University of New York (SUNY), \\ Binghamton, NY 13902-6000, USA
}

\section{ABSTRACT}

We present experimental observations of electron-beam irradiation induced diffusion and void formation confined in a one-dimensional two-segment copper-tin (Cu-Sn) nanowire system. The two segmented $\mathrm{Cu}-\mathrm{Sn}$ nanowires were synthesized by a layer-by-layer electrodeposition method using a nanoporous template. The structure and morphology of the nanowires were characterized by SEM, STEM, TEM, EDS and elemental mapping. Under e-beam irradiation, it is found that the nanowire surface oxide effectively confined the movement of metallic copper and tin atoms and the void regions were formed due to anisotropic diffusion rates and/or Sn melting. It is also seen that $\mathrm{Cu} / \mathrm{Sn}$ alloy nanoparticles were formed on the oxide layer in the void regions. Such 1-D cylindrical nanowire structure presents an ideal system for understanding dimensionally confined transport phenomena and alloy formation at the nanometer scale.

KEYWORDS: Nanowires, Lead-Free Solders, Copper-Tin, Diffusion, Nanoelectronics Assembly.

\section{INTRODUCTION}

Soldering is widely used in electronics assembly and packaging. Recently, due to the environmental and hêlth concerns of lead, traditional tin-lead $(\mathrm{Sn}-\mathrm{Pb})$ solders are being phased out and lead-free solders are becoming increasingly popular. Furthermore, the demand for highperformance interconnects in advanced packaging has caused the dramatic scaling down of the electronics packaging feature size from microscale to nanoscale. Among several techniques that have been proposed to address the nanoscale assembly and packaging, nanosolder-based joining and interconnection techniques provide great potential for forming robust joints between nanostructures and integrating nanocomponents into a functional device or complex system. ${ }^{1-3}$ In order to meet the green manufacturing requirements, nano-soldering based on lead-free solder materials would certainly hold greatest advantage. However, many fundamental issues involving nano-soldering have not been addressed, such as surface wetting on various substrates, diffusion, intermetallic formation, nanojoint

\footnotetext{
*Author to whom correspondence should be addressed.

Email: Zhiyong_Gu@uml.edu

Received: 25 August 2011

Accepted: 21 January 2012
}

strength and reliability, etc. The lack of understanding in these important issues hinders the development of this promising nano-joining technique. In many conventional applications involving copper-based circuitry, the interdiffusion between solder and copper substrate has been studied extensively for its significant role in $\mathrm{Cu}-\mathrm{Sn}$ intermetallic formation. ${ }^{4-6}$ However, the diffusion and resulting structure evolution in nanoscale $\mathrm{Cu}-\mathrm{Sn}$ systems have not been studied yet. Solder nanowire is an ideal model system for studying the solder diffusion at the nanoscale.

Electron beam irradiation, due to the strong energy of electron beam, has been widely used to induce phase transformation, ${ }^{7}$ shape transformation, ${ }^{8}$ and structure transformation. ${ }^{9}$ In this report, we present experimental investigation of one-dimensional diffusion in a lead-free $\mathrm{Cu}-\mathrm{Sn}$ two-segment nanowire system induced by electron beam irradiation in a transmission electron microscope (TEM). Using the e-beam irradiation, rather than by the traditional heating treatment, allows us to study the local interfacial transport and reactions in individual nanowires. Our observations show that both $\mathrm{Cu}$ and $\mathrm{Sn}$ have fast interdiffusion under e-beam irradiation, which leads to void formation, called 'Kirkendall voids', near the $\mathrm{Cu}-\mathrm{Sn}$ interface and around both ends of the nanowires. A diffusion and void formation mechanism is proposed based on our experimental observations. 


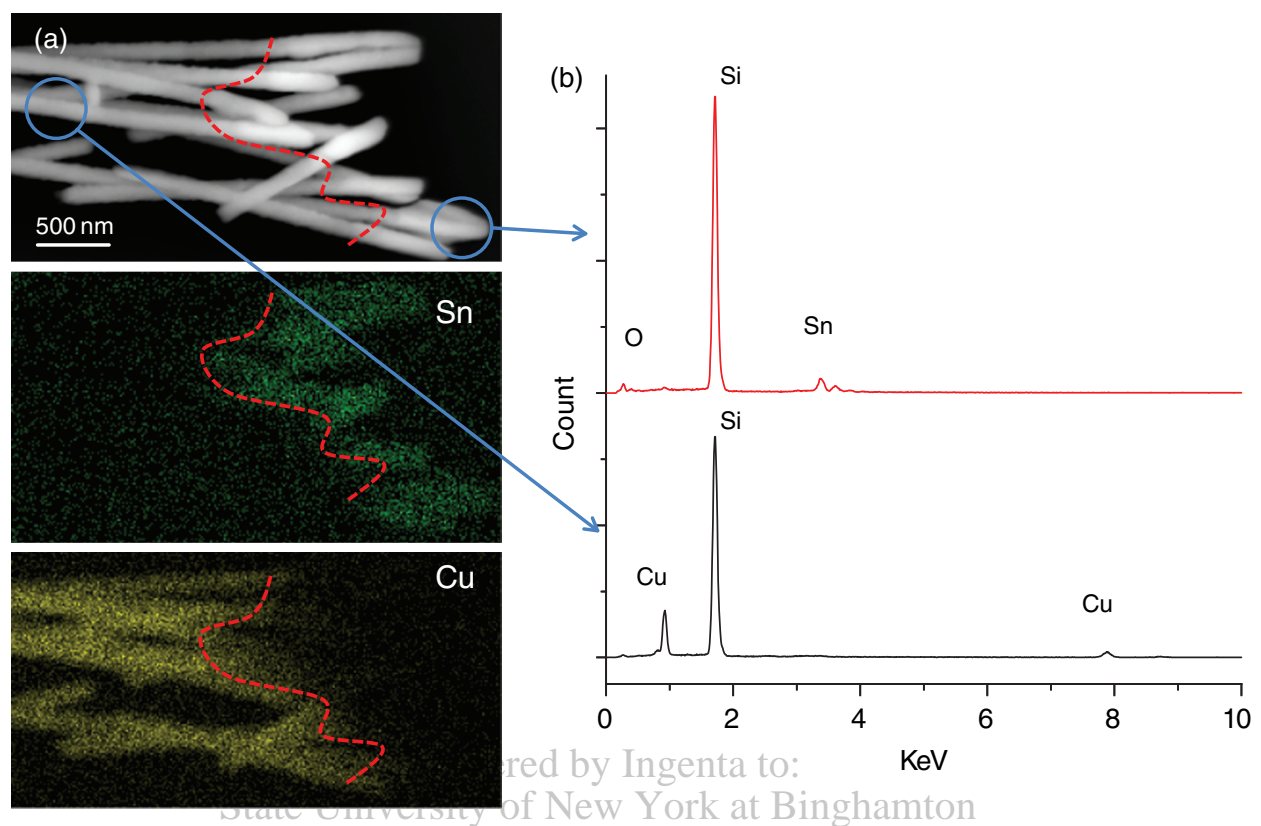

Fig. 1. (a) SEM image of as-fabricated $\mathrm{Cu}-\mathrm{Sn}$ two-segment nanowires at backscattered mode and EDS mapping for each segment; (b) spot mode EDS spectra for Sn-rich segment and Cu-rich segment from Figure 1(a).012 14:10:08

\section{EXPERIMENTAL DETAILS}

The $\mathrm{Cu}-\mathrm{Sn}$ two-segment nanowires were fabricated by sequential layer-by-layer electrodeposition method using a polycarbonate nanoporous membrane (Whatman) at room temperature. The average diameter of the nanopores was
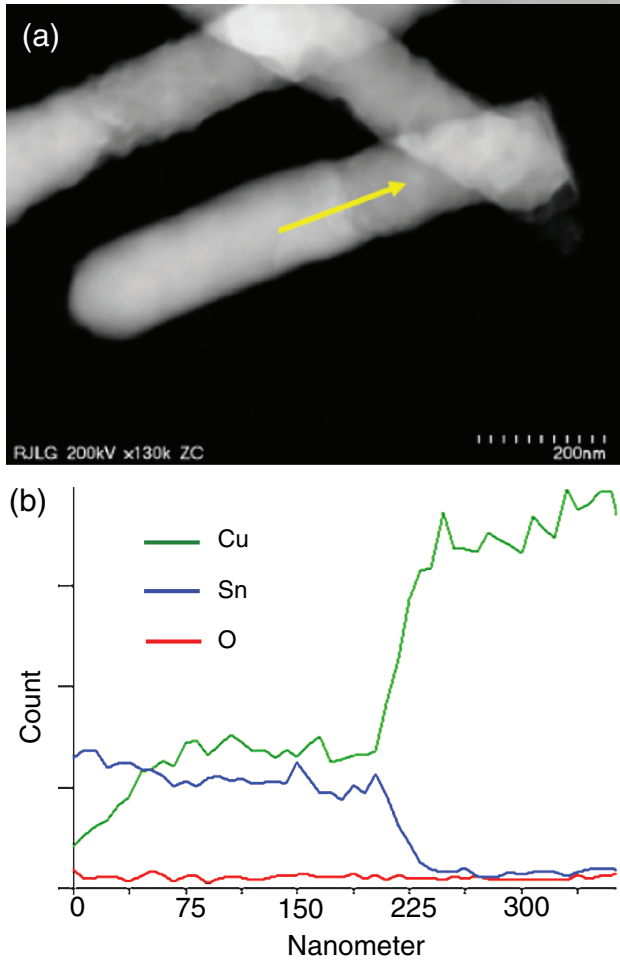

$50 \mathrm{~nm}$ (nominal value). The template has a gradual diameter increase from the surface to the inside channels. $\mathrm{Cu}$ was deposited first using a commercial $\mathrm{Cu}$ plating electrolyte (Cu U-bath RTU, Technic, Inc.) with the current controlled at $2 \mathrm{~mA} / \mathrm{cm}^{2}$. After $\mathrm{Cu}$ plating, the Sn layer was electroplated by commercial Sn plating electrolyte
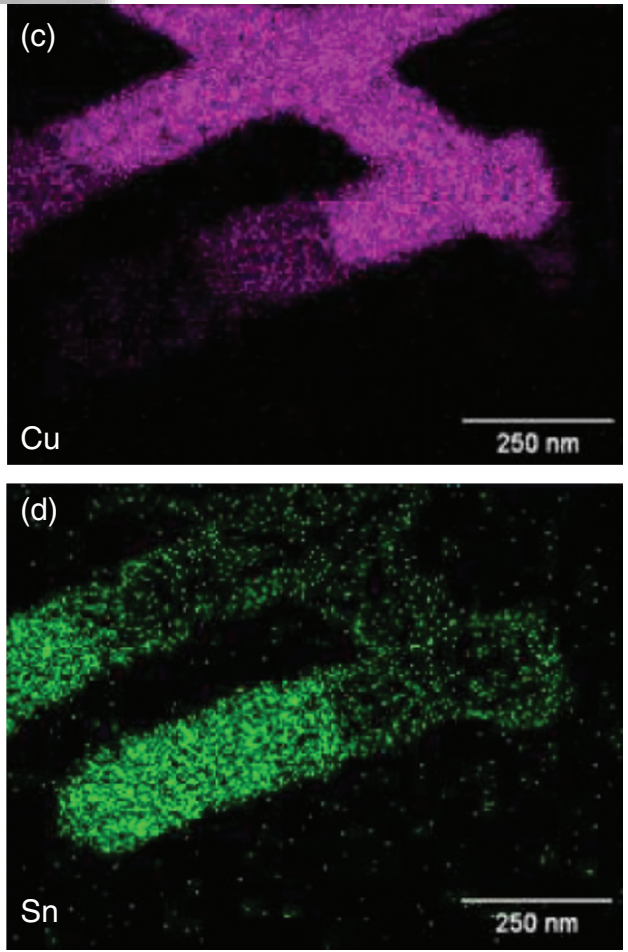

Fig. 2. (a) Z-contrast STEM image of $\mathrm{Cu}-\mathrm{Sn}$ nanowires at interface; (b) line profiles of copper, tin and oxygen elements across the interface as indicated by the arrow shown in Figure 2(a); (c-d) the elemental mapping of $\mathrm{Cu}(\mathrm{c})$ and $\mathrm{Sn}(\mathrm{d})$. 
(Sn Concentrate with make-up solutions, Technic, Inc.) with the current controlled at $18 \mathrm{~mA} / \mathrm{cm}^{2}$. After the electroplating, the polycarbonate membrane was dissolved in dichloromethane to release the nanowires into the solvent. The free-standing nanowires were cleaned and stored as a suspension in ethanol. The experimental setup and detailed information can be found from the previous publication. ${ }^{10}$ The as fabricated $\mathrm{Cu}-\mathrm{Sn}$ two segment nanowires were characterized by a JEOL-7401 field-emission scanning electron microscopy (FE-SEM) equipped with energy dispersive X-ray spectroscopy (EDS). The instrument was operated at $15 \mathrm{keV}$ under backscattered mode to get better imaging contrast in different segments.

TEM samples were prepared by depositing $\mathrm{Cu}-\mathrm{Sn}$ segmented nanowire samples (dispersed in ethanol) on a lacey carbon film supported on a Mo grid (SPI Supplies). A Mo grid was employed because its EDS spectrum is featureless near the $\mathrm{Cu}$ and $\mathrm{Sn} \mathrm{K}, \mathrm{L}$ lines used in the quantitative elemental analysis. TEM imaging and EDS spectroscopy were performed on a JEOL $2100 \mathrm{~F}$ electron microscope equipped with an Oxford INCA $30 \mathrm{~mm}^{2}$ atmospheric thin window (ATW) detector; the instrument was operated at $200 \mathrm{keV}$. All micrographs were acquired by a GIF TRIDIEM post-column energy filter (Gatan Inc.) and analyzed using DigitalMicrograph (Gatan Inc.) software. Z-contrast Scanning TEM images (STEM) and EDS spectra were also obtained on a Hitachi HD-2300A.

\section{RESULTS AND DISCUSSION}

$\mathrm{Cu}$ and $\mathrm{Sn}$ two segments were fabricated in the form of nanowires for the diffusion couple study. Figure 1 shows SEM images of as-synthesized $\mathrm{Cu}-\mathrm{Sn}$ nanowires with EDS characterization. The two segments can be clearly identified from the backscattered mode SEM image, as shown in Figure 1(a), and the red color dashed lines indicate the possible $\mathrm{Cu}-\mathrm{Sn}$ interfaces from the contrast difference. The brighter segment corresponds to the Sn-rich segment which has a higher atomic number than the darker region with the $\mathrm{Cu}$-rich segment. From the EDS elemental mapping image shown in Figure 1(a), it is easy to distinguish the two different elements at each location, which is consistent with contrast feature in the backscattered mode SEM image. Green and yellow areas delineated by the dashed line in the SEM image correspond to the tin and copper segments, respectively. It can be seen that the yellow signal crosses the interface with less intensity, suggesting a certain amount of copper atoms may have already migrated to the neighboring tin segment around the interfacial region during fabrication. The spot mode EDS spectra were collected from both the $\mathrm{Cu}$-rich and $\mathrm{Sn}$-rich segments (close to each end, as indicated by the circles in the SEM image of Fig. 1(a)). From Figure 1(b), the EDS results show that the $\mathrm{Cu}$-rich segment and Sn-rich segment contain larger than $99 \%$ purity of $\mathrm{Cu}$ or $\mathrm{Sn}$ in each segment, which confirms that there were two distinct segments. It is believed that no significant interdiffusion occurred under the low e-beam energy irradiation from the SEM. The big $\mathrm{Si}$ peak is from the background of the Si wafer, which was used to prepare the samples. There are two major factors that could contribute to the interface diffusion that may have occured during the electroplating process: electric field assisted ion diffusion and thermal diffusion. Since the $\mathrm{Cu}$ layer was deposited first, it is possible that a certain amount of top layer $\mathrm{Cu}$ atoms slowly diffused into the neighboring Sn layer during the tin electroplating.

The interface area was further studied through the STEM and EDS mapping. Figure 2(a) shows the $Z$ contrast STEM image of the $\mathrm{Cu}-\mathrm{Sn}$ nanowire at the $\mathrm{Cu}-\mathrm{Sn}$ interface area. Similar as Figure 1(a), the brighter contrast part represents Sn-rich segment and the darker contrast represents $\mathrm{Cu}$-rich segment. There are three nanowires in the image, with one nanowire ( $\mathrm{Cu}$ segment) lying on the top of the other two nanowires (on the $\mathrm{Cu}$ segments). An EDS line scan was performed across one nanowire interface as shown in Figure 2(b). An abrupt change in the $\mathrm{Cu}$ and $\mathrm{Sn}$ concentration is observed at the interface between the copper and tin segments, which is consistent with the abrupt contrast change across the interface as shown in Figure 2(a). The EDS line scan also reveals that almost
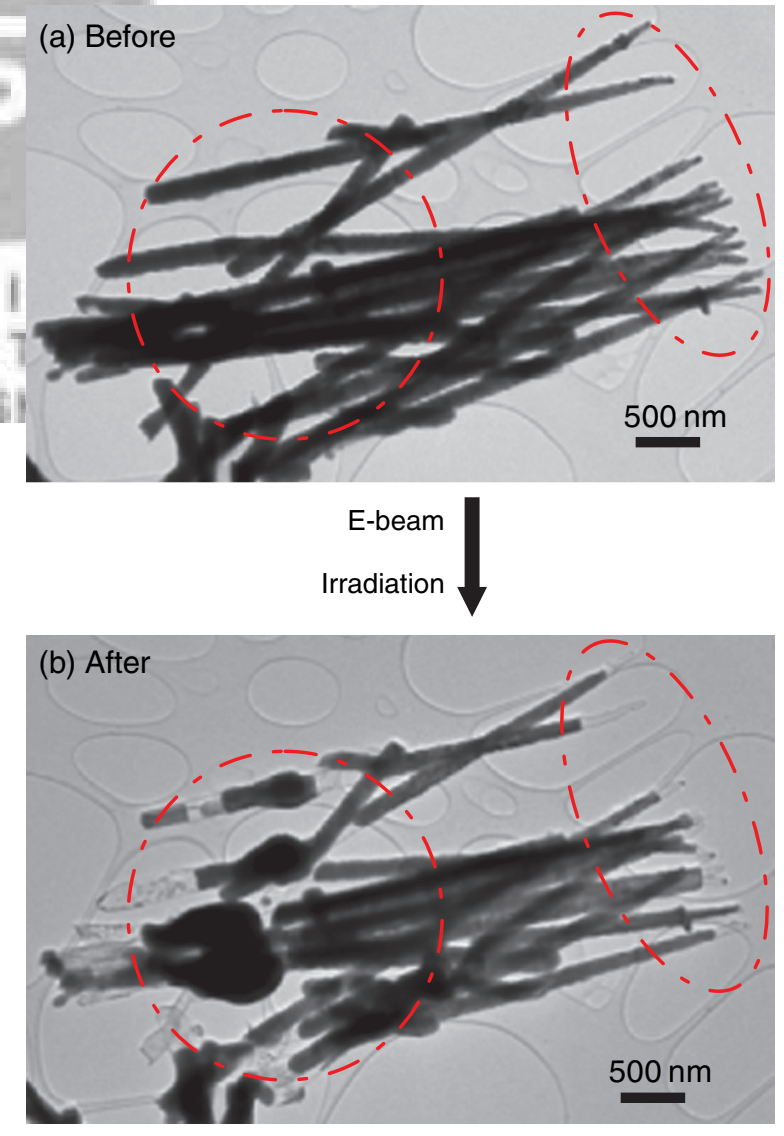

Fig. 3. TEM images showing the shape transformation of $\mathrm{Cu}-\mathrm{Sn}$ two segmented nanowires (a) before and (b) after the e-beam irradiation. 
no $\mathrm{Sn}$ was detected in the $\mathrm{Cu}$ segment. However, a certain amount of $\mathrm{Cu}$ was observed in the $\mathrm{Sn}$ segment, where the $\mathrm{Cu}$ concentration was constant for $\sim 150 \mathrm{~nm}$ away from the interface, indicating the formation of a $\sim 150 \mathrm{~nm}$ $\mathrm{Cu}-\mathrm{Sn}$ intermetallic phase. After this interfacial layer, $\mathrm{Cu}$ element gradually decreases towards the end of the $\mathrm{Sn}$ segment. The elemental mapping of the $\mathrm{Cu}$ (Fig. 2(c)) and Sn (Fig. 2(d)) also reveals a $\sim 200 \mathrm{~nm}$ region that contains both $\mathrm{Cu}$ and $\mathrm{Sn}$, showing the interfacial region. However, there is no significant tin signal shown in the $\mathrm{Cu}$ rich region beyond the interface, as shown in Figure 2(d). These results are consistent with SEM and EDS mapping result in Figure 1.

In order to investigate the effect of e-beam irradiation on the evolution of the mictrostructure and composition in the nanowires, several $\mathrm{Cu}-\mathrm{Sn}$ nanowires suspended on the TEM grid were selected with regular imaging electron beam illumination. Figure 3 shows the morphology change of the nanowires after the e-beam irradiation. The nanowires were uniform in size before the e-beam irradiation. Due to the template limitation, the end of the $\mathrm{Cu}$ segment has a sharp tip (smaller diameter). After the e-beam irradiation, there were voids formed at the tips of the $\mathrm{Cu}$-segments, but most copper were confined and kept in the nanowire shape. However, there was significant morphology transformation on the Sn-rich segment and big bulges formed. Large translucent voids were formed near the interface and on both $\mathrm{Cu}$-rich and $\mathrm{Sn}$-rich segments. These voids, called "Kirkendall void," formed because of the difference in diffusion rates between two components in a diffusion couple. ${ }^{11}$ With an increase in the e-beam exposure time, the void area would enlarge. The outer shell of voids is copper oxide or tin oxide which may have formed during the nanowire fabrication and storage. On the other hand, local tin melting may also contribute to the void formation around the interface. The melting of $\mathrm{Sn}$ under electron-beam irradiation may be caused by a temperature rise due to electron thermal spikes in the nanowire and poor thermal conduction away. ${ }^{12}$ On the other hand, it has been reported that there is a quasi-melting phase distinct from the thermodynamic molten state existing in small particles where the particles are continuously fluctuating between different structures at temperature well below the melting point. ${ }^{13,14}$ Hence, although the thermodynamic melting point of $\mathrm{Sn}$ is as high as $232^{\circ} \mathrm{C}$, the melting under the e-beam can be observed at a relatively low temperature. This phenomenon initiated by e-beam irradiation in TEM has been reported in other systems such as $\mathrm{Pb}$ nanobelts, ${ }^{15} \mathrm{ZnS}$ nanowire, ${ }^{16}$ etc.

A higher resolution TEM image of a representative Sn-rich region of the nanowire after the e-beam irradiation is shown in Figure 4. Most of the Sn element diffused into the $\mathrm{Cu}$-rich region and a void area confined in an oxide shell was formed. The dark tip shown on the top of the Sn-rich segment was confined at the end which may be due to relative higher surface tension trapped. From the EDS data and elemental composition analysis, those dark areas around both the tip and $\mathrm{Cu}-\mathrm{Sn}$ interface area were

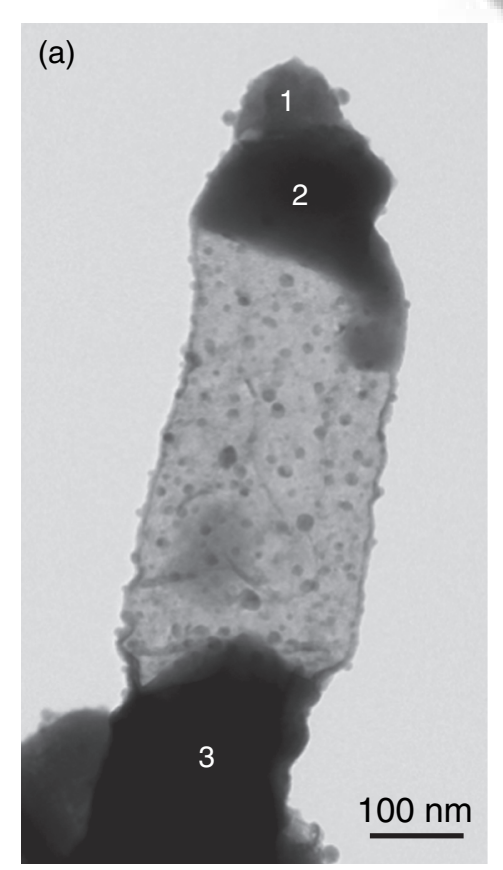

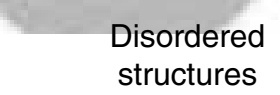
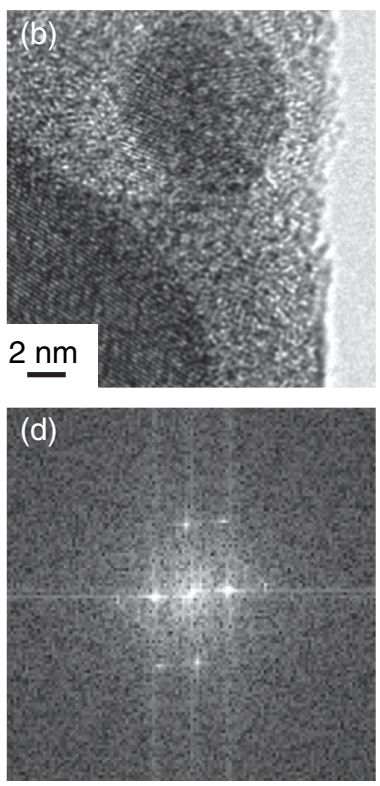

\section{E-beam Short-range \\ $\longrightarrow$ ordered structure}
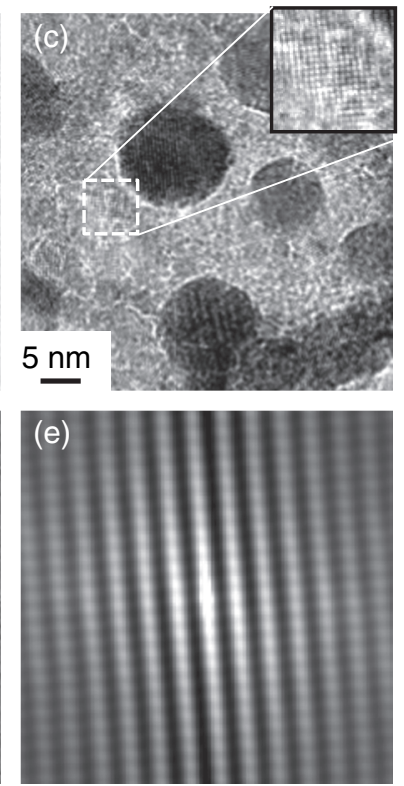

Fig. 4. (a) TEM images of Sn-rich region after e-beam irradiation; (b) HRTEM image of crystalline nanoparticles distributed on the disordered amorphous oxide shell in the void area; (c) HRTEM image of the reorganized larger crystalline nanoparticles and short-range ordered structure (inset image) formed on the oxide shell after the e-beam irradiation; (d) Fast Fourier transformed pattern of the crystalline ordered nanoparticle in (c); (e) inverse fast Fourier transform image of (d). 
Table I. Composition analysis of the Sn-rich segment (Fig. 4(a)) after e-beam irradiation.

\begin{tabular}{lcc}
\hline Point & \multicolumn{3}{c}{ Element weight\% } \\
\hline & $\mathrm{Cu}$ & $\mathrm{Sn}$ \\
1 & 37.79 & 62.21 \\
2 & 19.46 & 80.54 \\
3 & 51.02 & 48.98 \\
\hline
\end{tabular}

confirmed as $\mathrm{Cu} / \mathrm{Sn}$ alloy compounds with various ratios, as shown in Table I. The large amount of $\mathrm{Cu}$ ratio confirmed the diffusion of $\mathrm{Cu}$ atoms into the $\mathrm{Sn}$-rich regions under e-beam irradiation. From the corresponding HRTEM images, many nanoparticles were formed after the irradiation and were randomly distributed on the amorphous oxide shell surface. From Figures 4(b)-(c), it is interesting to observe that the nanoparticles were e-beam sensitive, since the focused nanoparticles tended to transform into better crystalline structure after longer e-beam irradiation. Some of the amorphous area with disordered structure would also transform into short-range ordered structure which were enlarged and shown in Figure 4(c) inset. The EDS results reveal that the nanoparticles are intermetallic rather than metal oxide. It is possible that the metallic nanoparticles were formed after atomic diffusion and then e-beam reduction/re-organization. ${ }^{17}$ Figures $4(\mathrm{~d})-(\mathrm{e})$ are the fast Fourier transform (FFT) and inverse fast Fourier transform (IFFT) images of the crystalline nanoparticles in Figure 4(c), showing the identification of the diffraction spots.

In Figure 5, the $\mathrm{Cu}$-rich region with void area was characterized by HRTEM imaging. The $\mathrm{Cu}$-rich region does not show melting behavior due to its high melting point, but the atomic diffusion can be clearly observed under the e-beam irradiation as shown in both Figures 3(b) and 5(b). Figure 5(c) shows the interface region of the

$$
\text { State University of New York at Binghamton }
$$
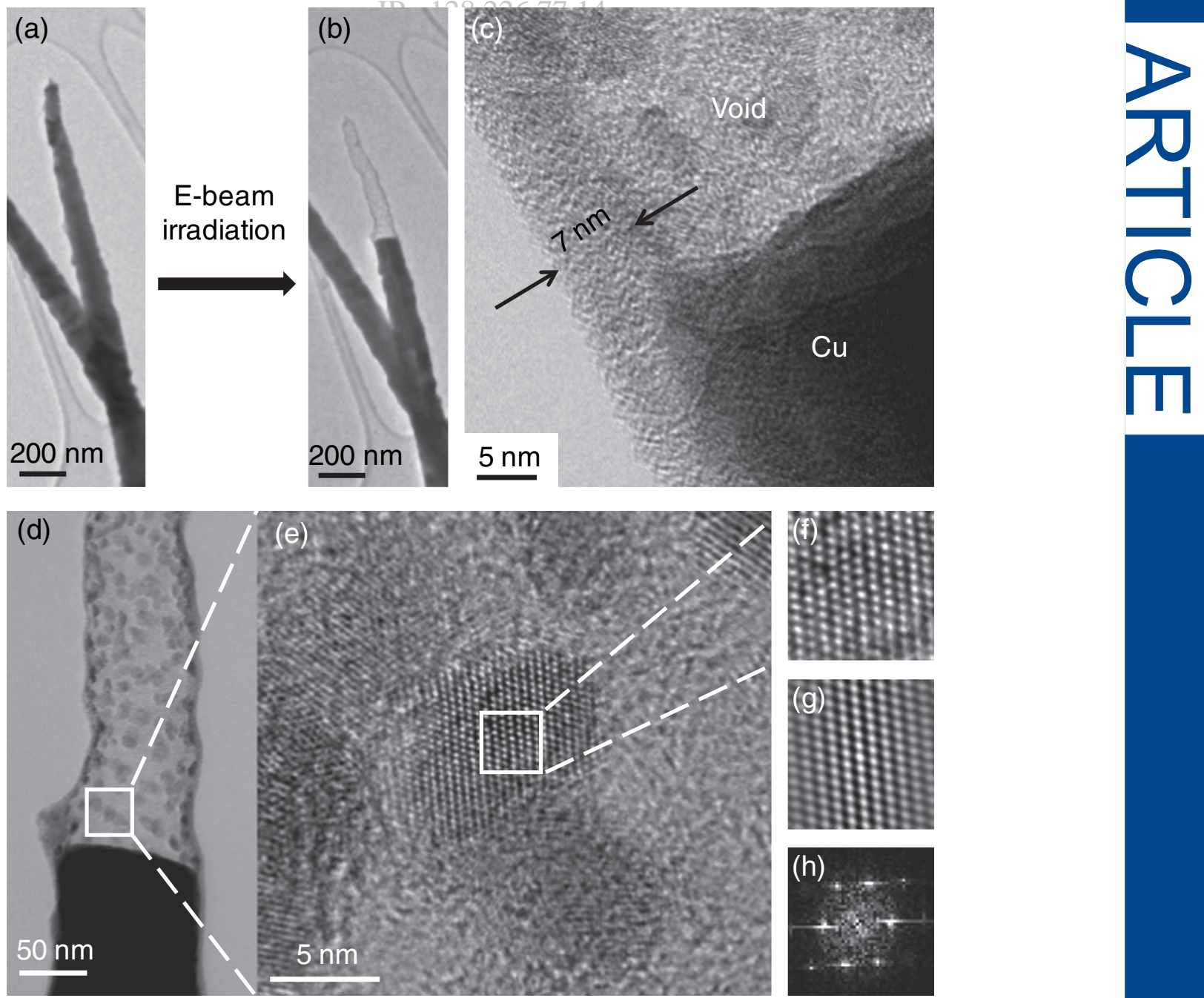

Fig. 5. TEM images of Cu-rich segment (a) before and (b) after e-beam irradiation; (c) HRTEM image of oxide shell of Cu segment with thickness measurement; (d) zoom in HRTEM image of Figure 5(b) with nanoparticles distributed on the copper oxide layer; (e) HRTEM image of crystalline ordered nanoparticles; (f) HRTEM in the selected square area in (e); (g) filtered HRTEM image of (f); (h) fast Fourier transformed image of (g). 
(1)

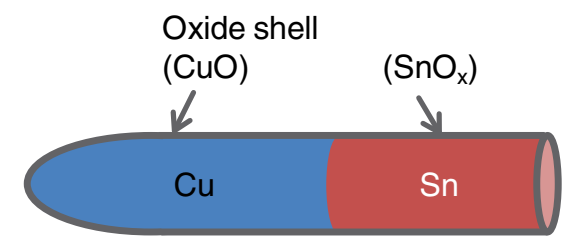

(2)

void

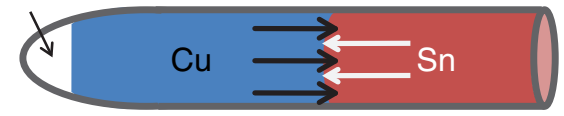

Atomic migration

(3)

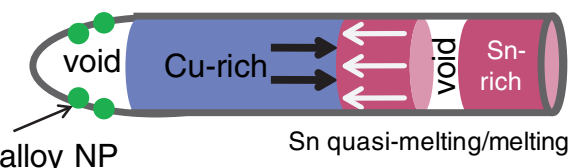

$\mathrm{Cu} / \mathrm{Sn}$ alloy NP

(4)

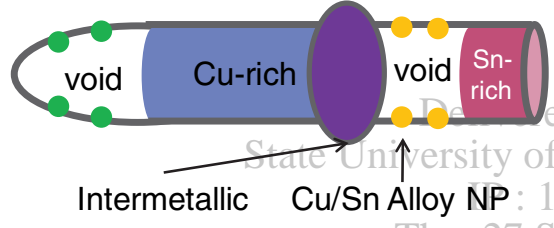

Thut, 27 Sep 20

Fig. 6. Schematic of proposed diffusion and void formation mechanism.
(1) original nanowire before e-beam irradiation; (2) anisotropic atomic diffusion in both $\mathrm{Cu}$-rich and $\mathrm{Sn}$-rich regions; (3) with the e-beam irradiation increasing, Sn-rich region reached quasi-melting/melting state with much faster diffusion into $\mathrm{Cu}$-rich region along the axial direction and the void area appeared; (4) intermatellic compounds grew to prevent further diffusion between $\mathrm{Cu}$ and $\mathrm{Sn}$.

amorphous copper oxide layer in the void area, neighboring with the metallic copper segment. Same as the Sn-rich segment, the $\mathrm{Cu}$-rich segment contains an oxide layer of about $7 \mathrm{~nm}$ thickness which can effectively confine the $\mathrm{Cu}$ diffusion along the axial direction under the e-beam irradiation. Nanoparticles distributed in the void area in Figure 5(d) had variable composition, similar to those in the Sn-rich void area by corresponding EDS analysis. The HRTEM image of nanoparticles distributed on the amorphous copper oxide shell is shown in Figure 5(e) and the crystalline lattice is enlarged and shown in Figure 5(f). The Figures 5(g)-(h) are the filtered HRTEM image and FFT of squared area in Figure 5(e), which are in excellent correlation and confirms the crystal structure of the nanoparticles.

Based on experimental observations, a possible $\mathrm{Cu}-\mathrm{Sn}$ diffusion and void formation mechanism is proposed as shown in Figure 6. At the beginning, due to the fact that copper has a smaller atomic size, the short time e-beam irradiation initiated fast copper diffusion into the $\mathrm{Sn}$-rich region. It has been reported that the magnitude of the diffusivity of copper in tin is orders of magnitude greater than that of tin in copper, ${ }^{18}$ which resulted in the copper atom migration and the Kirkendall void formation at the end of nanowire in the $\mathrm{Cu}$-rich segment. With an increase of the e-beam exposure time, tin was activated and reached the quasi-melting/melting state. The melting rate was much faster than the diffusion rate, ${ }^{19}$ and thus most of tin diffused into the $\mathrm{Cu}$-rich region along the axial direction under the tin oxide layer confinement and formed intermetallic compounds. Under further intensive e-beam irradiation, the $\mathrm{Cu} / \mathrm{Sn}$ alloy particles were formed and distributed on the amorphous oxide shell and then reorganized to form larger particles with better crystalline structure. The amorphous area would also transform into short-range ordered crystalline structure. Once the intermetallic layer is formed, the diffusion rate will dramatically decrease and further diffusion will be prevented. From this point of view, the e-beam induced irradiation on the $\mathrm{Cu}-\mathrm{Sn}$ diffusion system is consistent with the thermal treatment method..$^{20,21}$

\section{CONCLUSION}

In summary, two segmented $\mathrm{Cu}-\mathrm{Sn}$ nanowires were successfully synthesized by a sequential electrodeposition method using a nanoporous template. The diffusion and void formation of $\mathrm{Cu}-\mathrm{Sn}$ segmented nanowires induced by e-beam irradiation in a TEM have been studied. The metallic copper and tin embedded in the oxide shell were sensitive to the e-beam irradiation, resulting in the atomic diffusion, quasi-melting/melting of tin, and void formation. Alloy nanoparticles were formed on the oxide shell in the void regions. The diffusion was confined along the onedimensional structure due to the thin surface oxide layer.

Acknowledgments: We are grateful for the financial support from the $3 \mathrm{M}$ Non-Tenured Faculty Grant (ZG). This research was also partially supported by a grant from the NSF (DMR-0804892) (JY). The authors thank the Peterson Institute of NanoScience and Engineering (PINSE) at University of Pittsburgh and the R. J. Lee

Group for access of facility.

\section{References and Notes}

1. Z. Gu, H. Ye, D. Smirnova, D. Small, and D. H. Gracias, Small 2, 225 (2006).

2. F. Gao and Z. Gu, Nanotechnology 21, 115604 (2010).

3. Q. Cui, F. Gao, S. Mukherjee, and Z. Gu, Small 5, 1246 (2009).

4. A. Kar, M. Ghosh, A. K. Ray, and R. N. Ghosh, Mat. Sci. Eng. A 459, 69 (2007).

5. S. Li and C. Basaran, Comp. Mater. Sci. 47, 71 (2009).

6. S. Kang and A. Sarkhel, J. Electron. Mater. 23, 701 (1994).

7. X. Huang, S. Li, Y. Huang, S. Wu, X. Zhou, S. Li, C. L. Gan, F. Boey, C. A. Mirkin. and H. Zhang, Nat. Commun. 2, 292 (2011).

8. B. Wang, Y. H. Yang, and G. W. Yang, Nanotechnology 17, 5916 (2006).

9. K. H. An, K. A. Park, J. G. Heo, J. Y. Lee, K. K. Jeon, S. C. Lim, C. W. Yang, Y. S. Lee, and Y. H. Lee, J. Am. Chem. Soc. 125, 3057 (2003).

10. F. Gao, S. Mukherjee, Q. Cui, and Z. Gu, J. Phys. Chem. C 113, 9546 (2009).

11. Y. Yin, R. M. Rioux, C. K. Erdonmez, S. Hughes, G. A. Somorjai, and A. P. Alivisatos, Science 304,711 (2004). 
12. T. Yokota, M. Murayama, and J. M. Howe, Phys. Rev. Lett. 91, 265504 (2003).

13. P. M. Ajayan and L. D. Marks, Phys. Rev. Lett. 60,585 (1988).

14. P. M. Ajayan and L. D. Marks, Phys. Rev. Lett. 63,279 (1989).

15. Z. W. Pan, Z. R. Dai, and Z. L. Wang, Appl. Phys. Lett. 80, 309 (2002).

16. B. Liu, Y. Bando, M. Wang, C. Zhi, X. Fang, C. Tang, M. Mitome, and D. Golberg, J. Appl. Phys. 106, 034302 (2009).

17. Y. Komuro and Y. Matsumoto, J. Phys. Chem. C 115, 6618 (2011).
18. B. F. Dyson, T. R. Anthony, and D. Turnbull, J. Appl. Phys. 38, 3408 (1967).

19. M. Mouas, J. G. Gasser, S. Hellal, B. Grosdidier, A. Makradi, and S. Belouettar, EPJ Web of Conferences 15, 01013 (2011).

20. K. Zeng, R. Stierman, T.-C. Chiu, D. Edwards, K. Ano, and K. N. Tu, J. Appl. Phys. 97, 024508 (2005).

21. C.-C. Pan, C.-H. Yu, and K.-L. Lin, Appl. Phys. Lett. 93, 061912 (2008).

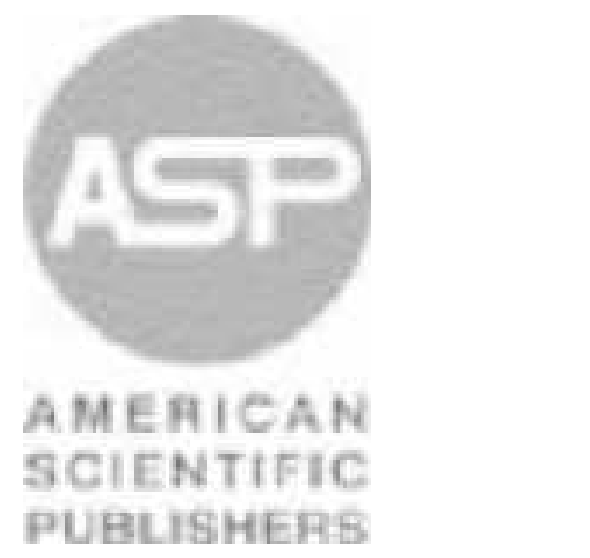

